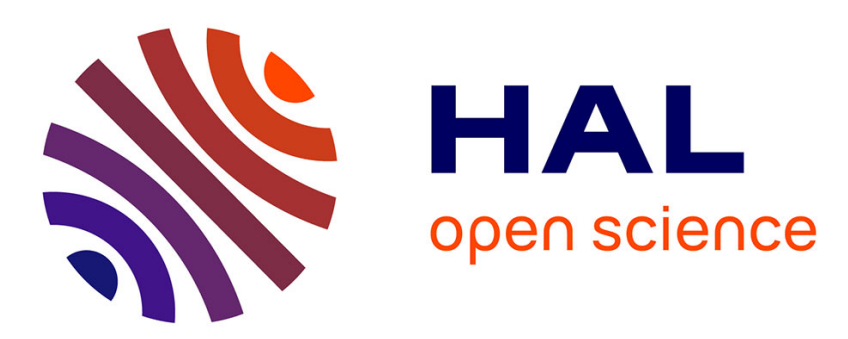

\title{
Stability of Sampled-data Control Systems under Aperiodic Sampling and Input Saturation
}

\author{
Mirko Fiacchini, João Manoel Gomes da Silva Jr
}

\section{To cite this version:}

Mirko Fiacchini, João Manoel Gomes da Silva Jr. Stability of Sampled-data Control Systems under Aperiodic Sampling and Input Saturation. CDC 2018 - 57th IEEE Conference on Decision and Control, Dec 2018, Miami, FL, United States. hal-01904488

\section{HAL Id: hal-01904488 \\ https://hal.univ-grenoble-alpes.fr/hal-01904488}

Submitted on 25 Oct 2018

HAL is a multi-disciplinary open access archive for the deposit and dissemination of scientific research documents, whether they are published or not. The documents may come from teaching and research institutions in France or abroad, or from public or private research centers.
L'archive ouverte pluridisciplinaire HAL, est destinée au dépôt et à la diffusion de documents scientifiques de niveau recherche, publiés ou non, émanant des établissements d'enseignement et de recherche français ou étrangers, des laboratoires publics ou privés. 


\title{
Stability of Sampled-data Control Systems under Aperiodic Sampling and Input Saturation
}

\author{
Mirko Fiacchini and João Manoel Gomes da Silva Jr.
}

\begin{abstract}
This work proposes a new approach to asses stability of sampled-data controlled linear systems under aperiodic sampling and subject to input saturation. From an impulsive representation of the system and considering a partition of the interval between two successive sampling instants, it is shown that the discrete-time dynamics of the closed-loop system can be described by a difference inclusion. A general Lyapunovbased result allowing to conclude about the local stability of the sampled-data system is derived. Thus, considering the particular case of quadratic functions, a constructive condition in terms of linear matrix inequalities (LMIs) is proposed to compute estimates of the region of attraction of the nonlinear closed-loop system.
\end{abstract}

\section{INTRODUCTION}

The digital control implementation paradigm brought the need of dealing with sampled signals and to consider simultaneously continuous and discrete-time dynamics in the control-loop. Considering periodic sampling policies, a large amount of works have been tackling this problem along the last decades (see, for instance, [1]-[6]). More recently, motivated by the implementation of digital control loops over networks (networked control) [7], the study of sampleddata control under aperiodic sampling has been the focus of new results in the field. In this context we can cite some different approaches to tackle the problem. In [8], [9] an uncertain discrete-time model, which is embedded onto a polytopic model from the use of exponential matrices is considered. In [10]-[12] the effects of the aperiodic sampling are modeled through norm bounded uncertainties. The timedelay approach, which addresses the aperiodic sampling by means of a time-varying delay on the control signal and considers an analysis based on Lyapunov-Krasovskii functionals has been proposed in [13] and further developed in [14] and [15]. A mixed continuous and discrete-time approach has been proposed in [16], [17] through the concept of looped-functionals and adapted to the case of impulsive systems in [18]. In [19] polyhedral Lyapunov functions are employed to obtain necessary and sufficient stability conditions and a constructive method for testing the stability. Finally, [20] deals with the stability verification problem using reachability analysis.

M. Fiacchini is with Univ. Grenoble Alpes, CNRS, Gipsa-lab, F-38000 Grenoble, France. mirko.fiacchiniegipsa-lab.fr

J.M. Gomes da Silva Jr. is with the Department of Automation and Energy Systems, Universidade Federal do Rio Grande do Sul (UFRGS), Porto Alegre, Brazil. jmgomes@ufrgs.br

This work has been supported by Project STICAmSud CoDySCo2 18STIC-01 (grant CAPES, Brazil no. 88881.143275/2017-01). J.M. Gomes da Silva Jr. is also supported by CNPQ, Brazil (grants PQ-305979/2015-9 and Univ-422992/2016-0)
On the other hand, due to actuator physical limitations, control signal saturation is an ubiquitous feature. Motivated by this, a large amount of works consider the problems of stability analysis and stabilization of systems with input saturation (see, for example, the books [21] and [22] for a general overview). In this vein, many approaches to ensure global or regional (local) asymptotic stability of the closedloop systems under saturating control laws have been proposed considering either continuous or discrete-time systems. Considering the periodic sampling case, the problem of assessing stability of a continuous-time plant controlled by a discrete-time dynamic output feedback control law has been addressed for instance, in [23]. In [24], the authors extended the design of discrete-time anti-windup loops to the sampled-data case with constant period by using the approach proposed in [2], in order to transpose the problem into a discrete-time framework. Aperiodic sampling in the presence of control saturation is addressed in [17] and [25] considering a looped-functional approach.

In this paper a new approach to analyse the stability of aperiodic sampled-data control, explicitly considering the possibility of control signal saturation, is proposed. The approach is based on the representation of the aperiodic sampled-data system by an impulsive system, where the control value is updated only at the sampling instants from the sampled value of the state. Taking into account that the continuous dynamics between two successive sampling instants is linear and considering a partition of the intersampling interval, it is shown that the discrete-time evolution of the system can be modeled by a difference inclusion. Based on this setup, a general Lyapunov-based condition is derived to assess the stability of the closed-loop system. This condition basically ensures that a Lyapunov function is strictly decreasing between sampling instants with a rate that is bigger than its possible expansion in the intersampling interval. Thus, considering quadratic Lyapunov function candidates and a polytopic differential inclusion representation of the saturation term, a construtive result, in form of linear matrix inequalities (LMIs), is proposed to compute estimates of the region of attraction of the origin of the nonlinear sampled-data controlled system. The approach is illustrated by a numerical example.

The paper is organized as follows. In section II an impulsive system representation for the sampled-data controlled system is presented and the stability assessment problem is formally stated. In Section III, from a suitable partition of the allowable interval between two successive samples, a difference inclusion describing the behavior of the discrete- 
time dynamics is proposed. Section IV is devoted to the main results of the paper. An illustrative example is provided in section V. Some concluding remarks end the paper.

Notation. $\mathbb{R}$ is the set of real numbers and $\mathbb{N}$ the set of natural ones. We denote $\mathbb{N}_{n} \triangleq\{i \in \mathbb{N}: 1 \leq i \leq n\}$. For any function $x$ defined on $\mathbb{R}^{n}$ we denote $x\left(t^{-}\right) \triangleq \lim _{\tau \mapsto t, \tau<t} x(\tau)$ if the limit exists. $\partial S$ and $\operatorname{int}(S)$ denote the boundary and the interior of a set $S$, respectively. $M_{(i)}$ and $v_{(i)}$ denote, respectively, the ith row of the matrix $M$ and the ith component of the vector $v .\|\cdot\|_{p}$ denotes the $p$-norm. $\sigma_{\max }(A)$ denotes the maximal real part of the eigenvalues of matrix $A$.

\section{Problem Formulation}

Consider the continuous-time plant described by the following linear model:

$$
\dot{x}_{p}(t)=A_{p} x_{p}(t)+B_{p} u(t)
$$

where $x_{p} \in \mathbb{R}^{n_{p}}$ and $u \in \mathbb{R}^{m}$ represent the state and the input of the plant, respectively. Matrices $A_{p}$ and $B_{p}$ have appropriate dimensions and are supposed to be constant. We assume that the control signal is constrained in magnitude, i.e.

$$
u \in \mathscr{U}=\left\{u \in \mathbb{R}^{m}:\|u\|_{\infty} \leq 1\right\} .
$$

From the input constraints, we consider that a saturating sampled-data control law given by

$$
u(t)=\operatorname{sat}\left(K_{p} x_{p}\left(t_{k}\right)\right), \quad \forall t \in\left[t_{k}, t_{k+1}\right)
$$

where $\operatorname{sat}(v): \mathbb{R}^{m} \rightarrow \mathbb{R}^{m}$ denotes a classical saturation function, whose elements $\operatorname{sat}_{(r)}(v), \forall r=1, \ldots, m$ are defined as

$$
\operatorname{sat}_{(r)}(v)=\operatorname{sign}\left(v_{(r)}\right) \min \left\{v_{(r)}, 1\right\},
$$

is applied to the system, and $t_{k}$ denotes the $k$-th sampling instant. Thanks to the presence of a $\mathrm{ZOH}$, the control input is kept constant at the value computed from the value of $x_{p}(t)$ sampled at the instant $t=t_{k}^{-}$, for all $t \in\left[t_{k}, t_{k+1}\right)$.

We assume that there exist two positive scalars $\tau_{m} \leq \tau_{M}$ such that the difference between two successive sampling instants $\delta_{k}=t_{k+1}-t_{k}$ satisfies

$$
0<\tau_{m} \leq \delta_{k} \leq \tau_{M}, \quad \forall k \in \mathbb{N}
$$

The bounds $\tau_{m}$ and $\tau_{M}$ are supposed to be imposed by constraints on the digital hardware and a networked control implementation. In this last case, they can represent for instance a jitter on a nominal periodic sampling, induced by the communication protocol.

Note that assuming $\tau_{m}>0$ is sufficient to avoid Zeno solution and then that the sequence $\left\{t_{k}\right\}_{k \in \mathbb{N}}$ is an unbounded increasing sequence of positive scalars. The intersampling time $\delta_{k}$ can be variable, which allows to model an aperiodic sampling strategy. The particular case of periodic sampling corresponds to $\delta_{k}=\tau_{m}=\tau_{M}$ for all $k \in \mathbb{N}$. Given the interval $\Delta=\left[\tau_{m}, \tau_{M}\right]$ with $0<\tau_{m} \leq \tau_{M}$ and $t_{0}=0$, the set of sequences of admissible sampling instants is given by:

$$
\Theta(\Delta)=\left\{\left\{t_{k}\right\}_{k \in \mathbb{N}}: t_{k+1}=t_{k}+\delta_{k}, \delta_{k} \in \Delta, \quad \forall k \in \mathbb{N}\right\} .
$$

Then, for every $\mathscr{T} \in \Theta(\Delta)$, the system dynamics can be represented by the following impulsive system:

$$
\begin{aligned}
& \begin{cases}\dot{x}_{p}(t)=A_{p} x_{p}(t)+B_{p} u(t), & \forall t \in \mathbb{R}_{+}-\mathscr{T}, \\
\dot{u}(t)=0, & \forall t \in \mathscr{T},\end{cases} \\
& \left\{\begin{array}{l}
x_{p}(t)=x_{p}\left(t^{-}\right), \\
u(t)=\operatorname{sat}\left(K_{p} x_{p}\left(t^{-}\right)\right),
\end{array}\right. \\
& x_{p}(0)=x_{0} \in \mathbb{R}^{n_{p}}, \quad u(0)=\operatorname{sat}\left(K_{p} x_{0}\right) \in \mathbb{R}^{m},
\end{aligned}
$$

or, equivalently [26], by

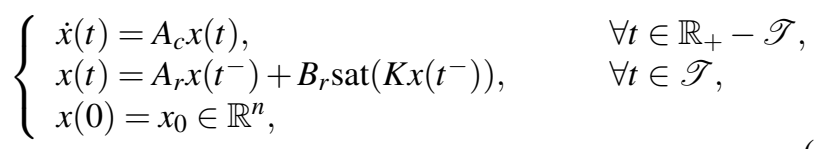

where $x=\left[\begin{array}{ll}x_{p}^{\prime} & u^{\prime}\end{array}\right]^{\prime} \in \mathbb{R}^{n}$, with $n=n_{p}+m$, is the overall system state and $A_{c}, A_{r} \in \mathbb{R}^{n \times n}$ and $B_{r} \in \mathbb{R}^{n \times m}$ are given as follows

$$
\begin{aligned}
& A_{c}=\left[\begin{array}{ll}
A_{p} & B_{p} \\
0 & 0
\end{array}\right], \quad A_{r}=\left[\begin{array}{ll}
I & 0 \\
0 & 0
\end{array}\right], \\
& B_{r}=\left[\begin{array}{l}
0 \\
I
\end{array}\right], \quad K=\left[\begin{array}{ll}
K_{p} & 0
\end{array}\right] \text {. }
\end{aligned}
$$

It should be noticed that, due to the saturation term the closed-loop system (6) is nonlinear. Hence, the global stability of the origin cannot be a priori guaranteed. Moreover, if matrix $A_{p}$ is not Hurwitz, the global stabilization is actually impossible [27]. In this case, since the analytical characterization of the region of attraction of the origin (RAO) is in general not possible, the idea is to estimate it through well-defined Lyapunov domains. These domains can therefore be seen as "safe" regions of operation.

In this paper we are particularly interested in the local asymptotic stability guarantee and the determination of estimates of the RAO for system (6). The problem we focus on can therefore be stated as follows.

Problem 1: Given the bounds $\tau_{m}$ and $\tau_{M}$ on the intersampling time:

a) provide conditions that allow to asses the local stability of the origin of the closed-loop system, considering an aperiodic sampling policy with $\delta_{k} \in\left[\tau_{m}, \tau_{M}\right], \forall k \in \mathbb{N}$;

b) once the local stability of the origin is ensured, determine an estimate $\mathscr{R}$ of the RAO.

\section{EQUIVALENT DISCRETE-TIME UNCERTAIN SYSTEM}

For given $x_{0}$ and $\mathscr{T} \in \Theta(\Delta)$ the evolution of the state $x$ between two successive sampling instants, i.e. for $t \in$ $\left[t_{k}, t_{k+1}\right)$, is continuous. Thus, since the dynamics is linear, it follows that:

$$
x(t)=e^{A_{c}\left(t-t_{k}\right)} x\left(t_{k}\right), \quad \forall t \in\left[t_{k}, t_{k+1}\right) .
$$

Hence, taking into account, from (5), that $x_{p}(t)=x_{p}\left(t^{-}\right)$and equations (6) and (8), the dynamics between two successive sampling instants is given by the following discrete-time equation

$$
\begin{aligned}
x\left(t_{k+1}\right) & =A_{r} e^{A_{c}\left(t_{k+1}-t_{k}\right)} x\left(t_{k}\right)+B_{r} \operatorname{sat}\left(K e^{A_{c}\left(t_{k+1}-t_{k}\right)} x\left(t_{k}\right)\right) \\
& =A_{r} e^{A_{c} \delta_{k}} x\left(t_{k}\right)+B_{r} \operatorname{sat}\left(K e^{A_{c} \delta_{k}} x\left(t_{k}\right)\right),
\end{aligned}
$$


where $\delta_{k}=t_{k+1}-t_{k} \in \Delta$. Thus, denoting $A(\delta)=A_{r} e^{A_{c} \delta}$ and $K(\delta)=K e^{A_{c} \delta}$, the problem of stability of the linear impulsive system (6) can be tackled by considering the following discrete-time nonlinear parametric uncertain system

$$
x\left(t_{k+1}\right)=A\left(\delta_{k}\right) x\left(t_{k}\right)+B_{r} \operatorname{sat}\left(K\left(\delta_{k}\right) x\left(t_{k}\right)\right), \quad \text { with } \delta_{k} \in \Delta .
$$

Consider now the partition of the interval $\left[\tau_{m}, \tau_{M}\right]$ in $J \in \mathbb{N}$ sub-intervals and define the set:

$$
\Delta_{J}=\left\{\tau_{m}+(j-1) \tau_{J}: j \in \mathbb{N}_{J}\right\}, \quad \tau_{J}=\frac{\tau_{M}-\tau_{m}}{J} .
$$

Since $\delta_{k} \in\left[\tau_{m}, \tau_{M}\right]$ and from (11), we have that for every $\delta_{k}$ there exist $d_{k} \in \Delta_{J}$ and $\tau_{k} \in\left[0, \tau_{J}\right]$ such that $\delta_{k}=d_{k}+\tau_{k}$ and it follows that:

$$
\begin{aligned}
& A\left(\delta_{k}\right)=A\left(d_{k}+\tau_{k}\right)=A_{r} e^{A_{c}\left(d_{k}+\tau_{k}\right)}=A\left(d_{k}\right) e^{A_{c} \tau_{k}}, \\
& K\left(\delta_{k}\right)=K\left(d_{k}+\tau_{k}\right)=K e^{A_{c}\left(d_{k}+\tau_{k}\right)}=K\left(d_{k}\right) e^{A_{c} \tau_{k}} .
\end{aligned}
$$

Thus, from (10), we can write that:

$$
x\left(t_{k+1}\right)=A\left(d_{k}\right) e^{A_{c} \tau_{k}} x\left(t_{k}\right)+B_{r} \operatorname{sat}\left(K\left(d_{k}\right) e^{A_{c} \tau_{k}} x\left(t_{k}\right)\right),
$$

with $d_{k} \in \Delta_{J}$ and $\tau_{k} \in\left[0, \tau_{J}\right]$. Then, given the state $x\left(t_{k}\right)=x_{k}$ at sampling instant $t_{k}$, the possible successors are given by the difference inclusion

$$
\begin{aligned}
x_{k+1} & \in\left\{A(d) e^{A_{c} \tau} x_{k}+B_{r} \operatorname{sat}\left(K(d) e^{A_{c} \tau} x\left(t_{k}\right)\right): d \in \Delta_{J},\right. \\
\tau & \left.\in\left[0, \tau_{J}\right]\right\}=\left\{A(d) y+B_{r} \operatorname{sat}(K(d) y): d \in \Delta_{J},\right. \\
y & \left.=e^{A_{c} \tau} x_{k}, \tau \in\left[0, \tau_{J}\right]\right\} .
\end{aligned}
$$

Hence, defining the following set-valued maps

$$
\begin{aligned}
& \mathscr{F}(y)=\left\{A(d) y+B_{r} \operatorname{sat}(K(d) y): d \in \Delta_{J}\right\} \subseteq \mathbb{R}^{n}, \\
& \mathscr{G}(z)=\left\{e^{A_{c} \tau} z: \tau \in\left[0, \tau_{J}\right]\right\} \subseteq \mathbb{R}^{n},
\end{aligned}
$$

$\forall y, z \in \mathbb{R}^{n}$, then for every $y_{k} \in \mathscr{G}\left(x_{k}\right)$ we have that $x_{k+1} \in$ $\mathscr{F}\left(y_{k}\right)$, i.e. the dynamics in (14) are also given by the difference inclusions

$$
x_{k+1} \in \mathscr{F}\left(\mathscr{G}\left(x_{k}\right)\right) .
$$

Thus, the stability of the difference inclusion system (16) can be used to analyse the stability of the closed-loop system (1)(2) under asynchronous sampling with inter-sampling time bounded by $\tau_{m}$ and $\tau_{M}$.

\section{Stability Analysis}

\section{A. Lyapunov setup}

Let now $V(x): \mathbb{R}^{n} \rightarrow \mathbb{R}$ be a continuous Lyapunov candidate function, such that

$$
\mu_{1}\|x\|_{p}^{p} \leq V(x) \leq \mu_{2}\|x\|_{p}^{p}
$$

The level sets associated to $V(x)$, generically defined as

$$
\Omega_{c}=\left\{x \in \mathbb{R}^{n}: V(x) \leq c\right\}
$$

are compact sets containing the origin in their interior for all $c>0$.

Considering the function $V(x)$ and the difference inclusion in (16), a sufficient condition for the asymptotic stability of the origin of the closed-loop system (1)-(2) and the characterization of estimates of the RAO are given in the following theorem.
Theorem 1: Consider a set $\mathscr{D}$ containing the origin in its interior. Suppose that there exists a scalar $\lambda \in(0,1)$ such that the following conditions hold:

a) $V\left(y^{+}\right) \leq \lambda V(y)$ for all $y \in \mathscr{D}$ and $y^{+} \in \mathscr{F}(y)$, with $\mathscr{F}(\cdot)$ as defined in (15);

b) $\dot{V}(x)<\alpha V(x)$ along the trajectories of system

$$
\dot{x}(t)=A_{c} x(t),
$$

with $\alpha=\frac{1}{\tau_{J}} \ln \left(\frac{1}{\lambda}\right)$. Then the origin is locally asymptotically stable for the aperiodic sampled-data saturated control system (1)-(2) and the sets $\Omega_{c}$, defined as in (18), such that $\Omega_{c / \lambda} \subseteq \mathscr{D}$, are contained in its domain of attraction.

Proof: Denote $x\left(t_{k}\right)=x_{k}$ and consider $V\left(x_{k}\right)=c$. We first prove that $V\left(x_{k+1}\right)<V\left(x_{k}\right)$, for every $x_{k}$ in a neighborhood of the origin with $x_{k+1}$ given by (16). Recall that for all $y_{k} \in \mathscr{G}\left(x_{k}\right)$ there exists $\tau \in\left[0, \tau_{J}\right]$ such that $y_{k}=e^{A_{c} \tau} x_{k}, e^{A_{c} \tau} x_{k}$ being the solution of (19) at time $t_{k}+\tau$. Then from condition $\mathrm{b}$ ) and since $\alpha=\frac{1}{\tau_{J}} \ln \left(\frac{1}{\lambda}\right)>0$, one obtains:

$$
\begin{array}{ll}
V\left(y_{k}\right)=V\left(x_{k}\right)<e^{\alpha \tau_{J}} V\left(x_{k}\right)=\frac{1}{\lambda} V\left(x_{k}\right)=\frac{c}{\lambda}, & \text { if } \tau=0 \\
V\left(y_{k}\right)<e^{\alpha \tau} V\left(x_{k}\right) \leq e^{\alpha \tau_{J}} V\left(x_{k}\right)=\frac{1}{\lambda} V\left(x_{k}\right)=\frac{c}{\lambda}, & \text { if } \tau>0
\end{array}
$$

Thus, for every $x_{k} \in \mathbb{R}^{n}$ such that $V\left(x_{k}\right)=c$ one has that $\mathscr{G}\left(x_{k}\right) \subseteq \Omega_{c / \lambda}$. If moreover $\Omega_{c / \lambda} \subseteq \mathscr{D}$, then condition a) applies for every element $y_{k} \in \mathscr{G}\left(x_{k}\right)$ and then

$$
V\left(y_{k}^{+}\right) \leq \lambda V\left(y_{k}\right)<V\left(x_{k}\right)=c,
$$

for all $x_{k+1}=y_{k}^{+} \in \mathscr{F}\left(y_{k}\right)=\mathscr{F}\left(\mathscr{G}\left(x_{k}\right)\right)$, which means that $V\left(x_{k+1}\right)<V\left(x_{k}\right)$ for all $x_{k+1} \in \mathscr{F}\left(\mathscr{G}\left(x_{k}\right)\right)$, or, equivalently, that $x_{k+1} \in \operatorname{int}\left(\Omega_{c}\right)$, provided $x_{k} \in \partial \Omega_{c}$. From the continuity of $V(x)$ and the fact that the origin is in the interior of $\mathscr{D}$, there exists $c>0$ such that $\Omega_{c / \lambda} \subseteq \mathscr{D}$ and hence $x_{k} \rightarrow 0$ as $k \rightarrow+\infty$, provided that $x_{0}=x(0) \in \Omega_{c}$.

Consider now $t \in\left[t_{k}, t_{k+1}\right)$. From (19) and (17), it follows:

$$
\begin{aligned}
V(x(t)) & \leq \mu_{2}\|x(t)\|_{p}^{p}=\mu_{2}\left\|e^{A_{c}\left(t-t_{k}\right)} x\left(t_{k}\right)\right\|_{p}^{p} \\
& \leq \mu_{2} \max _{\tau \in\left[\tau_{m}, \tau_{M}\right]}\left\|e^{A_{c} \tau}\right\|_{p}^{p}\left\|x\left(t_{k}\right)\right\|_{p}^{p},
\end{aligned}
$$

which implies uniform stability and that $V(x(t)) \rightarrow 0$ as $x\left(t_{k}\right)=x_{k} \rightarrow 0$. Thus we conclude that then the origin is asymptotically stable for the aperiodic sampled-data saturated control system (1)-(2). Moreover, the sets $\Omega_{c}$ are contained in the domain of attraction if $\Omega_{c / \lambda} \subseteq \mathscr{D}$.

A similar result could have been found using the hybrid systems framework [28] instead of the considered impulsive one. In particular, the impulsive systems framework allows to cast the problem in terms of difference inclusions (see (16)), which is more suitable, in our opinion, for obtaining constructive stability conditions and to quantify the related conservatism. Actually, in case of absence of saturation, constructive necessary and sufficient conditions for stability based on the difference inclusions model have been obtained for instance in [19]. It should be noticed that, differently from a classical hybrid approach, conditions a) and b) in Theorem 1 do not regard directly the continuous and discrete dynamics in (6). The basic idea consists in ensuring the decreasing of the Lyapunov function between two jumps using 
the discrete-time differencial inclusion $\mathscr{F}(y)$, considering the uncertainty on the sampling instant through the set-valued $\operatorname{map} \mathscr{G}(z)$.

Remark 1: Note that, since $\alpha$ is supposed to be a positive scalar, condition b) in Theorem 1 does not impose a decreasing of the function $V$ in continuous-time. Actually, it ensures a bound on its potential increasing, which means that $A_{c}$ is not required to be Hurwitz. In this case, the verification of condition b) will require a sufficiently large $\alpha>0$.

\section{B. Testable Conditions}

In order to obtain testable conditions, in this section we apply Theorem 1 considering $V(x)$ as a quadratic function. This will allow to express conditions a) and b) as linear matrix inequalities (LMIs) and therefore to formulate convex optimization problems to determine estimates of the RAO of the system.

With this aim, the first step regards the choice of an appropriate and tractable model for the saturation term present in (15). We particularly consider the approach proposed in [29] and re-formulated as a polytopic inclusion in [22], which can be summarized in the following Lemma:

Lemma 1: Let $D_{i} \in \mathbb{R}^{m \times m}, i \in \mathbb{N}_{2^{m}}$ all possible different diagonal matrices whose diagonal elements are 1 or 0 and $\bar{D}_{i}=\left(I_{m}-D_{i}\right)$. For matrices $H_{i} \in \mathbb{R}^{m \times n}, i \in \mathbb{N}_{2^{m}}$, define the following sets:

$$
S\left(H_{i}, 1\right)=\left\{x \in \mathbb{R}^{n}:\left|H_{i(r)} x\right| \leq 1, \forall r \in \mathbb{N}_{m} \text { s.t. } \bar{D}_{i(r)} \neq 0\right\}
$$

If $x \in \bigcap_{i=1}^{2^{m}} S\left(H_{i}, 1\right)$ thus it follows that there exist scalars $0 \leq$ $\gamma_{i} \leq 1$, with $\sum_{i=1}^{2^{m}} \gamma_{i}=1$, such that

$$
\operatorname{sat}(K x)=\sum_{i=1}^{2^{m}} \gamma_{i}\left(D_{i} K+\bar{D}_{i} H_{i}\right) x
$$

Based on Lemma 1 and the conditions in Theorem 1 with a quadratic function $V(x)=x^{\prime} P x, P=P^{\prime}>0$, we can now state a constructive condition to address Problem 1.

Theorem 2: If there exist a matrix $W=W^{\prime}>0, W \in \mathbb{R}^{n \times n}$, matrices $G_{d, i} \in \mathbb{R}^{m \times n}$ with $d \in \Delta_{J}, i \in \mathbb{N}_{2^{m}}$ and scalars $\lambda \in$ $(0,1)$ and $\alpha=\frac{1}{\tau_{J}} \ln \left(\frac{1}{\lambda}\right)>\max \left\{2 \sigma_{\max }\left(A_{c}\right), 0\right\}$, satisfying the following LMIs

$$
\begin{gathered}
{\left[\begin{array}{cc}
\lambda W & W\left(A(d)+B_{r} D_{i} K(d)\right)^{\prime}+G_{d, i}^{\prime} \bar{D}_{i} B_{r}^{\prime} \\
\star & W
\end{array}\right] \geq 0,} \\
{\left[\begin{array}{cc}
W & G_{d, i(r)}^{\prime} \\
\star & 1
\end{array}\right]>0,} \\
\forall d \in \Delta_{J}, i \in \mathbb{N}_{2^{m}}, \quad i \in \mathbb{N}_{2^{m}}, \forall r \in \mathbb{N}_{m} \text { s.t. } \bar{D}_{i(r)} \neq 0 \\
W A_{c}^{\prime}+A_{c} W-\alpha W<0
\end{gathered}
$$

then, for all $x(0)=x_{0} \in \mathscr{E}\left(W^{-1}, \lambda\right)=\left\{x \in \mathbb{R}^{n} ; x^{\prime} W^{-1} x \leq\right.$ $\lambda\}$, it follows that the corresponding trajectory of the sampled-data system (1)-(2), with $\delta_{k}$ satisfying (3), converges asymptotically to the origin.
Proof: Consider $V(x)=x^{\prime} P x$ with $P=P^{\prime}>0, P=W^{-1}$. Using convexity arguments, applying Schur's complement to (23) and left and right multiplying the resulting inequality respectively by $y^{\prime} P$ and $P y$, it follows that:

$$
y^{\prime} \tilde{A}(d)^{\prime} P \tilde{A}(d) y-\lambda y^{\prime} P y \leq 0, \quad \forall d \in \Delta_{J}, i \in \mathbb{N}_{2^{m}}, \quad \forall y \neq 0,
$$

with $\tilde{A}(d)=A(d)+B_{r} \sum_{i=1}^{2^{m}} \gamma_{i}\left(D_{i} K(d)+\bar{D}_{i} H_{d, i}\right)$ and $H_{d, i}=$ $G_{d, i} P$. From Lemma 1 , it follows that $V\left(y^{+}\right) \leq \lambda V(y)$, for all $y^{+} \in \mathscr{F}(y)$, i.e. condition a) of Theorem 1 is satisfied, provided $y \in \bigcap_{i=1}^{2^{m}} S\left(H_{d, i}, 1\right)$, for all $d \in \Delta_{J}$.

Now applying Schur's complement to (24) and left and right multplying the resulting inequality by $y^{\prime} P$ and and $P y$, it follows that

$$
\left|H_{d, i(r)} y\right|^{2}<y^{\prime} P y
$$

which ensures that $\mathscr{E}\left(W^{-1}, 1\right)=\left\{y \in \mathbb{R}^{n} ; y^{\prime} W^{-1} y \leq\right.$ $1\} \subseteq S\left(H_{d, i}, 1\right)$, for every $i \in \mathbb{N}_{2^{m}}$ and all $d \in \Delta_{J}$, or equivalently, $\mathscr{E}\left(W^{-1}, 1\right) \subseteq \bigcap_{i=1}^{2^{m}} S\left(H_{d, i}, 1\right)$, for all $d \in \Delta_{J}$.

On other hand, note that if (25) holds, it follows that item b) of Theorem 1 is verified with $V(x)=x^{\prime} P x$. At this point note that (25) can be verified if and only if the eigenvalues of $A_{c}$ have real part strictly smaller than $\alpha / 2$. Thus, considering $\alpha>\max \left\{2 \sigma_{\max }\left(A_{c}\right), 0\right\}$ is needed to ensure the feasibility of inequality (25).

Hence, provided that $x(0)=x_{0} \in \mathscr{E}\left(W^{-1}, \lambda\right)$, we have

$$
y=\mathscr{G}\left(x_{0}\right) \in \mathscr{E}\left(W^{-1}, 1\right) \subseteq \bigcap_{d \in \Delta_{J}} \bigcap_{i=1}^{2^{m}} S\left(H_{d, i}, 1\right)
$$

and condition a) of Theorem 1 is indeed verified with $\mathscr{D}=$ $\bigcap_{d \in \Delta_{J}} \bigcap_{i=1}^{2^{m}} S\left(H_{d, i}, 1\right)$, which concludes the proof.

\section{Optimization Problems}

Given the bounds $\tau_{m}$ and $\tau_{M}$ on $\delta_{k}$, we can use the conditions of Theorem 2 to compute regions of guaranteed stability for the sampled-data closed-loop system, i.e. estimates of the region of attraction of the origin. Actually, provided $x(0) \in \mathscr{E}\left(W^{-1}, \lambda\right)$, conditions of Theorem 1 guarantee that the corresponding trajectory converges asymptotically to the origin.

Thus, the idea is to maximize region $\mathscr{E}\left(W^{-1}, \lambda\right)$, considering some size criterion. For instance, the maximization of the minor axis of the set can be considered through the following optimization problem:

$$
\begin{aligned}
& \min _{W, G_{d, i}, \varepsilon} \varepsilon \\
& \quad \text { subject to: } \\
& \quad(23),(24),(25), \\
& \quad\left[\begin{array}{cc}
\varepsilon I & I \\
\star & W
\end{array}\right]>0 .
\end{aligned}
$$

The last constraint in problem (27), ensures that the maximal eigenvalue of $W^{-1}$ is smaller than $\varepsilon$. Hence, the minimization of $\varepsilon$ corresponds to the maximization of the 
minor axis of the ellipsoid $\mathscr{E}\left(W^{-1}, \lambda\right)$. Other size criteria, such as the volume maximization or the maximization of the set in certain directions can also be easily considered (see [22]).

Note that problem (27) is associated to a given partition of the interval $\left[\tau_{m}, \tau_{M}\right]$. In order to find a suitable partition, the following algorithm is proposed:

Algorithm:

- Step 1: Fix $\lambda$

- Step 2: Initialize $J$

- Step 3: Compute $\tau_{J}=\frac{\tau_{M}-\tau_{m}}{J}$

- Step 4: Fix $\alpha=\frac{1}{\tau_{J}} \ln \left(\frac{1}{\lambda}\right)$ and solve (27):

If (27) is not feasible, $J \leftarrow J+1$ and go to Step 3 .

Otherwise stop.

Remark 2: Note that, as pointed out in the proof of Theorem 2, a necessary condition for the feasibility of (25) is that $\alpha=\frac{1}{\tau_{J}} \ln \left(\frac{1}{\lambda}\right)>\max \left\{2 \sigma_{\max }\left(A_{c}\right), 0\right\}$. Hence, for a fixed $\lambda$, we should have

$$
\tau_{J}<\frac{1}{\max \left\{2 \sigma_{\max }\left(A_{c}\right), 0\right\}} \ln \left(\frac{1}{\lambda}\right)
$$

which implies, from (11), that the initialization of $J$ in Step 3 of the Algorithm should satisfy:

$$
J>\left(\tau_{M}-\tau_{m}\right) \frac{\max \left\{2 \sigma_{\max }\left(A_{c}\right), 0\right\}}{\ln \left(\frac{1}{\lambda}\right)}
$$

Remark 3: It is worth noticing that the region $\mathscr{E}\left(W^{-1}, \lambda\right)$ is defined in the space of $x=\left[\begin{array}{ll}x_{p}^{\prime} & u^{\prime}\end{array}\right]^{\prime}$. On the other hand, note that the actual initial states $x_{p}(0)$ and $u(0)$ are coupled by the relation:

$$
u(0)=\operatorname{sat}\left(K_{p} x_{p}(0)\right)
$$

Hence, the "safe" set of plant initial states, i.e. the set in the plant subspace such that $x(0)=\left[\begin{array}{ll}x_{p}(0) & u(0)^{\prime}\end{array}\right]^{\prime} \in$ $\mathscr{E}\left(W^{-1}, \lambda\right)$ is in fact described by the set

$\mathscr{X}_{p}=\left\{x_{p} \in \mathbb{R}^{n_{p}} ;\left[\begin{array}{c}x_{p} \\ \operatorname{sat}\left(K_{p} x_{p}\right)\end{array}\right]^{\prime} W^{-1}\left[\begin{array}{c}x_{p} \\ \operatorname{sat}\left(K_{p} x_{p}\right)\end{array}\right] \leq \lambda\right\}$,

Note that the region $\mathscr{X}_{p}$ corresponds to the level set of a piecewise quadratic function.

\section{NumERicAl EXAMPLE} [22]:

Consider the system (1)-(2) with the following matrices

$$
\begin{gathered}
A_{p}=\left[\begin{array}{cc}
0 & 1 \\
1 & 0
\end{array}\right] ; B_{p}=\left[\begin{array}{c}
0 \\
-5
\end{array}\right] ; \\
K_{p}=\left[\begin{array}{ll}
2.6 & 1.4
\end{array}\right]
\end{gathered}
$$

with the interval of admissible intersampling times given by $\Delta=[0.05,0.1]$.

In this case, for $\lambda=0.96$ a feasible solution for the optimization problem (27) is obtained with a partition of $\Delta$ in 14 sub-intervals (i.e. $J=14$ ), leading to:

$$
W^{-1}=P=\left[\begin{array}{ccc}
0.6364 & 0.2866 & -0.1454 \\
0.2866 & 0.2114 & -0.0875 \\
-0.1454 & -0.0875 & 0.0858
\end{array}\right]
$$

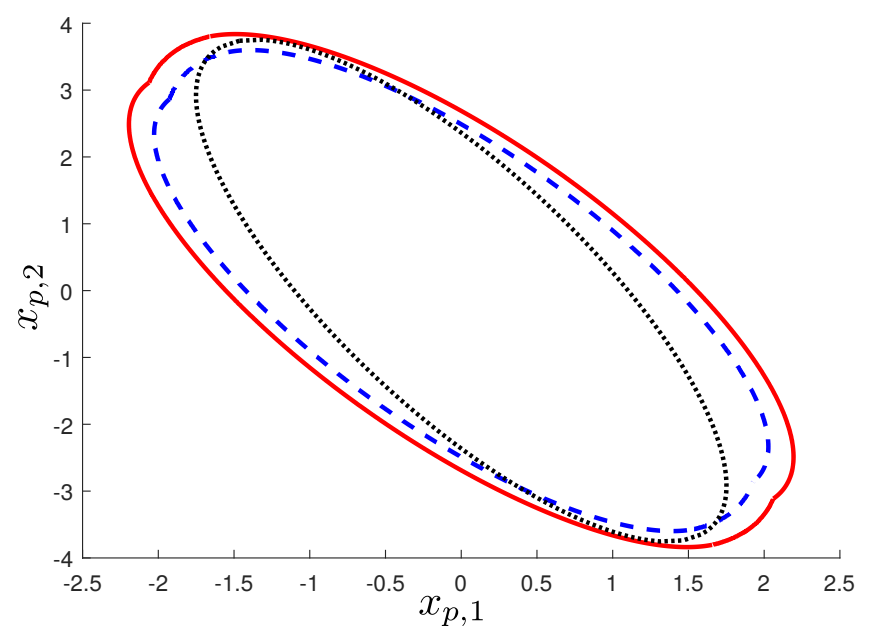

Fig. 1. region $\mathscr{X}_{p}$ for $\lambda=0.96$ (blue-dashed) and $\lambda=0.98$ (redcontinuous) and region obtained with the approach in [17] (black-dotted)

and the optimal $\varepsilon=0.8198$.

Considering now $\lambda=0.98$, a feasible solution for the optimization problem (27) is obtained for $J=27$, leading to:

$$
W^{-1}=P=\left[\begin{array}{ccc}
0.5341 & 0.2397 & -0.1242 \\
0.2397 & 0.1811 & -0.0752 \\
-0.1242 & -0.0752 & 0.0754
\end{array}\right]
$$

and the optimal $\varepsilon=0.6894$.

Note that for a smaller value of $\lambda$ (i.e. a more stringent requirement of contraction for the discrete-time trajectories) a larger value of $\varepsilon$ is obtained, denoting a reduction on the size of the domain. This is indeed verified in Figure 1 , where the regions of stability $\mathscr{X}_{p}$ (i.e. the estimates of the region of attraction) obtained for $\lambda=0.96$ and $\lambda=0.98$ are displayed. For comparison purposes, we also plotted the ellipsoidal domain obtained with the conditions proposed in [17], considering a looped-functional approach. It can be observed that the domain $\mathscr{X}_{p}$ for $\lambda=0.98$ includes the estimate generated from the result in [17]. It should also be observed that since $\mathscr{X}_{p}$ is piecewise quadratic, the region of stability obtained is nonconvex.

In Figure 2, several trajectories starting at the boundary of the region $\mathscr{X}_{p}$ (obtained for $\lambda=0.98$ ) and considering $\delta_{k}$ randomly chosen in the interval $\left[\begin{array}{ll}0.05 & 0.1\end{array}\right]$ are shown. As expected, the convergence of the trajectories to the origin is ensured showing that $\mathscr{X}_{p}$ is indeed included in its region of attraction.

\section{CONCLUSIONS}

In this paper, a new constructive approach has been presented to test the stability of a sampled-data controlled linear system under aperiodic sampling and saturating inputs. The method consists in computing a local Lyapunov function for the system with sampling instants belonging to a discrete subset of the admissible interval such that it is decreasing for all the possible sampling times. In particular, considering 


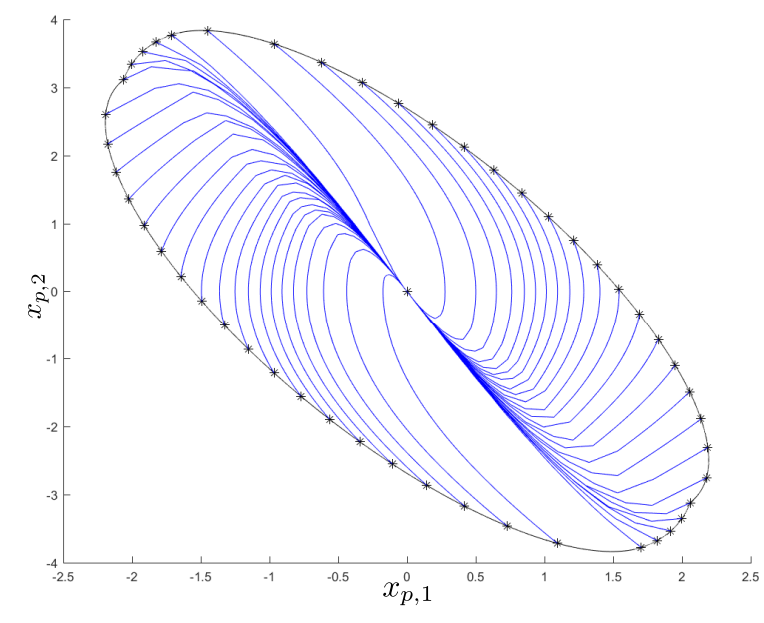

Fig. 2. Trajectories starting at the boundary of $\mathscr{X}_{p}$.

quadratic Lyapunov function candidates, the approach leads to convex conditions in form of LMIs.

As possible future work, we consider to take into account the stabilization problem, concerning the design of the state feedback matrix. Another point to be explored regards the use of more general Lyapunov functions as, for instance, polyhedral ones.

\section{REFERENCES}

[1] K. J. Aström and B. Wittenmark, Computer Controlled Systems. Theory and Design. Information and System Sciences Series, PrenticeHall, 1984.

[2] T. Chen and B. Francis, Optimal sampled-data control systems. Springer-Verlag, 1995.

[3] S.-T. Chung and J. Grizzle, "Sampled-data observer error linearization," Automatica, vol. 26, no. 6, pp. 997-1007, 1990.

[4] J. Grizzle and P. Kokotovic, "Feedback linearization of sampled-data systems," IEEE Trans. on Automatic Control, vol. 33, no. 9, pp. 857859, 1988.

[5] D. Nešić, A. Teel, and E. Sontag, "Formulas relating KL stability estimates of discrete-time and sampled-data nonlinear systems," Systems \& Control Letters, vol. 38, no. 1, pp. 49-60, 1999.

[6] D. Nešić and A. Teel, "A framework for stabilization of nonlinear sampled-data systems based on their approximate discrete-time models," IEEE Trans. on Automatic Control, vol. 49, no. 7, pp. 1103-1122, 2004.

[7] W. Zhang, M. Branicky, and S. Phillips, "Stability of Networked Control Systems," IEEE Control Systems Magazine, vol. 21(1), pp. 8499, 2001.

[8] M. Cloosterman, L. Hetel, N. V. D. Wouw, W. Heemels, J. Daafouz, and H. Nijmeijer, "Controller synthesis for networked control systems," Automatica, vol. 46, no. 10, pp. 1584-1594, 2010.

[9] M. Cloosterman, N. V. D. Wouw, W. Heemels, and H. Nijmeijer, "Stability of networked control systems with uncertain time-varying delays," IEEE Trans. on Automatic Control, vol. 54, no. 7, pp. 15751580, 2009.

[10] H. Fujioka, "Stability analysis of systems with aperiodic sample- andhold devices," Automatica, vol. 45, no. 3, pp. 771-775, 2009.

[11] C.-Y. Kao and H. Fujioka, "On stability of systems with aperiodic sampling devices," IEEE Trans. on Automatic Control, vol. 28, no. 3, pp. 2085-2090, 2013.

[12] Y. Oishi and H. Fujioka, "Stability and stabilization of aperiodic sampled-data control systems using robust linear matrix inequalities," Automatica, vol. 46, no. 8, pp. 1327-1333, 2010.
[13] E. Fridman, A. Seuret, and J.-P. Richard, "Robust sampled-data stabilization of linear systems: An input delay approach," Automatica, vol. 40, no. 8, pp. 1141-1446, 2004.

[14] E. Fridman, "A refined input delay approach to sampled-data control," Automatica, vol. 46, no. 2, pp. 421-427, 2010.

[15] P. Naghshtabrizi, J. Hespanha, and A. Teel, "Exponential stability of impulsive systems with application to uncertain sampled-data systems," Systems \& Control Letters, vol. 57, no. 5, pp. 378-385, 2008.

[16] A. Seuret, "A novel stability analysis of linear systems under asynchronous samplings," Automatica, vol. 48, no. 1, pp. 177-182, 2012.

[17] A. Seuret and J. M. Gomes da Silva Jr., "Taking into account period variations and actuator saturation in sampled-data systems," Systems \& Control Letters, vol. 61, pp. 1286-1293, 2012.

[18] C. Briat and A. Seuret, "A looped-functional approach for robust stability analysis of linear impulsive systems," Systems \& Control Letters, vol. 61, no. 10, pp. 980-988, 2012.

[19] M. Fiacchini and I.-C. Morărescu, "Constructive necessary and sufficient condition for the stability of quasi-periodic linear impulsive systems," IEEE Transactions on Automatic Control, vol. 61, no. 9, pp. 2512-2517, 2016.

[20] M. Al Khatib, A. Girard, and T. Dang, "Stability verification and timing contract synthesis for linear impulsive systems using reachability analysis," Nonlinear Analysis: Hybrid Systems, vol. 25, pp. 211-226, 2017.

[21] T. Hu and Z. Lin, Control systems with actuator saturation: analysis and design. Boston: Birkhauser, 2001.

[22] S. Tarbouriech, G. Garcia, J. M. Gomes da Silva Jr., and I. Queinnec, Stability and Stabilization of Linear Systems with Saturating Actuators. Springer, 2011.

[23] D. Dai, T. Hu, A. Teel, and L. Zaccarian, "Output feedback synthesis for sampled-data system with input saturation," in American Control Conference (ACC 2010), (Baltimore, USA), pp. 1797-1802, June 2010.

[24] G. Herrmann, M. Turner, and I. Postlethwaite, "Discrete-time and sampled-data anti-windup synthesis: stability and performance," International Journal of Systems Science, vol. 37, no. 2, pp. 91-113, 2006.

[25] J. M. Gomes da Silva Jr., I. Queinnec, A. Seuret, and S. Tarbouriech, "Regional stability analysis of discrete-time dynamic output feedback under aperiodic sampling and input saturation," IEEE Transactions on Automatic Control, vol. 61, pp. 4176-4182, Dec 2016.

[26] L. Hetel, J. Daafouz, S. Tarbouriech, and C. Prieur, "Stabilization of linear impulsive systems through a nearly-periodic reset," Nonlinear Analysis: Hybrid Systems, vol. 7, no. 1, pp. 4-15, 2013.

[27] H. J. Sussmann, S. D. Sontag, and Y. Yang, "A general result on the stabilization of linear systems using bounded controls," IEEE Transactions on Automatic Control, vol. 39, no. 12, pp. 2411-2425, 1994.

[28] R. Goebel, R. G. Sanfelice, and A. R. Teel, Hybrid Dynamical Systems: modeling, stability, and robustness. Princeton University Press, 2012.

[29] T. Alamo, A. Cepeda, D. Limón, and E. F. Camacho, "A new concept of invariance for saturated systems," Automatica, vol. 42, no. 9, pp. 1515-1521, 2006. 